\title{
LA-7532-MS
}

DR. 207

MASTER

Effect of Deviations from the Maxwell Distribution on Neutron Production in Laser Targets

임

to

$\frac{7}{\infty}$

$\stackrel{\frac{\Phi}{2}}{\frac{2}{5}}$ 
LA-7532-MS

Informal Report

UC-21

Issued: November 1978

\section{Effect of Deviations from the Maxwell Distribution on Neutron Production in Laser Targets}

D. B. Henderson

A. G. Petschek *

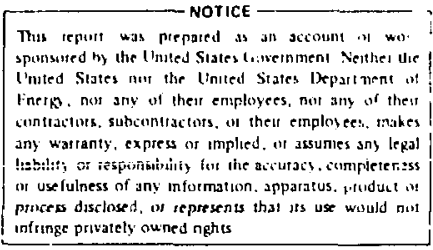

"Consuitant. 122 Piedra Loop, Los Alamos, NM 87544.

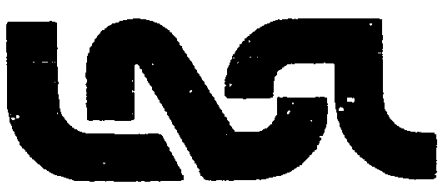




\title{
EFFECT OF DEVIATIONS FROM THE MAXWELL DISTRIBUTION
}

ON NEUTRON PRODUCTION IN LASER TARGETS

by

D. B. Henderson and A. G. Petschek

\begin{abstract}
Because of the brief duration of laser implosions and the small size of the peiliets, one may be concerned that the ions never reach a Maxweil distribution or that the tail is lost by diffusion. This might have a large effect on $\langle\sigma v\rangle$, which depends heavily on the tail. We have calculated the ion distribution and the DT <OV $\rangle$. Results are presented for the ratio of $\langle\sigma v\rangle$ for a monoenergetic isotropic distribution to that for a Maxwell distribution, for the rate of approach of $\langle\sigma v\rangle$ to the equilibrium value, and for the decay of $\langle\sigma v\rangle$ due to fast ion losses. The main effect in the last case is due to energy losses, not to non-Maxwellian distribution. The effect is substantially different than previously reported.
\end{abstract}

The thermonuclear burn of laser-fusion pellets may be usefully studied by consiueration of the burn of hot compressed microspheres. " Such microspheres may be thought of as being in the inner core of DT droplets that have been compressed and heated by iaser-diriven abiation of thie outer region of the pellet. ${ }^{2-3}$ From such studies we know that the fractional burnup $f_{\text {ro }}$ may be expected to follow from the fusion reactivity $\langle\sigma v\rangle$ as 1

$$
f_{r o}=\left(\frac{<\sigma v>}{8 C_{S} M_{i}}\right) \rho R
$$

for conditions in which $f_{\text {ro }}<<1$. In evaluating the expressions in Eq. (1), the density $\rho$, radius $R$, and the sound speed $C=\gamma\left(2 T / N_{j}\right)^{j / 2}$ are evaluated at the initial conditions. We use $\gamma=5 / 3$ and $M_{j}$ is the ion mass. For <ov> 
we use the average over the ion distributions,

$$
\langle\sigma v\rangle=\frac{1}{2} \int_{0}^{\infty} v_{1}^{2} d v_{1} \int_{0}^{\infty} v_{2}^{2} d v_{2} \int_{0}^{\infty} d \mu \iota_{1}\left(v_{1}\right) g_{2}\left(v_{2}\right) \sigma(v)
$$

where $g_{1}$ and $g_{2}$ are normalized distribution functions of the two-ion species, assumed isotropic. $\quad v$ is the relative velocity, given by

$$
v^{2}=v_{1}^{2}+v_{2}^{2}-2 v_{1} v_{2} u
$$

where $\mu$ in turn is the cosine of the angle between $v_{1}$ and $v_{2}$. For numerical integration it is most convenient to replace $\mu$ by $v$ in the integration to obtain

$$
\langle\sigma v\rangle=\frac{l}{2} \int_{0}^{\infty} g_{1}\left(v_{1}\right) d v_{1} \int_{0}^{\infty} g_{2}\left(v_{2}\right) d v_{2} \int_{\left|v_{1}-v_{2}\right|}^{v_{1}+v_{2}} v^{2} \sigma(v) d v
$$

and then to tabulate $\int_{0}^{w} v^{2} \sigma(v) d v$ so that only quadratures on $v_{1}$ ard $v_{2}$ need be done.

In the special case of $g_{1}$ and $g_{2}$ being Maxwell distributions, it is well known that the distribution of relative velocities is also a Maxwell distribution so that one can write

$$
\langle\sigma v\rangle_{\infty}=\int_{0}^{\infty} v \sigma(v) g(v) v^{2} d v .
$$

Justification of the use of a Maxwell distribution out to infinity requires thât the meañ free path för test ions at speed $v, \lambda(v)$, be smaller than dimensions of interest over ranges of $v$ important to the integration. Unfortunately the cross-section. $\sigma$, rises very rapidly with increasing $v$ (below the maximum near $100 \mathrm{keV}$, deuteron energy) so that important ranges of $v$ in the integration may correspond to energies well above the thermal energy in $g(v)$ and to very long mean free paths $\lambda(v)$.

If the distribution of relative velocities is Maxwellian and the energy dependence of the cross section is dominated by Gamow barrier penetration, one finds that the integrand of Eq. (5) has a maximum at a relative energy $6.8(\mathrm{keV})^{1 / 3}$ 
$(\mathrm{kT})^{2 / 3}$, that is at $6.8(\mathrm{keV} / \mathrm{kT})^{1 / 3}$ times $\mathrm{kT}$. The mean free path for $90^{\circ}$ deflection for a test ion, on the other hand, is ${ }^{4}$

$$
\lambda=M_{i}{ }^{2} v^{4} / 8 \pi ; n a^{4} \ln \Lambda .
$$

Substituting $E_{c}=M_{j} v^{2} / 2$ and $n=\rho / M_{j}$, this expression can be evaluated as

$$
E_{c}=559 \mathrm{keV}(\ln N / T 0)^{1 / 2}(\rho \lambda)^{1 / 2} \text {, }
$$

where $\ln \Lambda=10$ is typical, applying to a $3.0 \mu \mathrm{g}$ microsphere of $\mathrm{DT}$ at $\rho R=10^{-4}$, and $k T=1 \mathrm{keV}$. Thus, if we consider that test ions with $\lambda \cap R$ ought to diffuse out of the hot compressed core rapidly and be lost to the thermonuclear burn, we would expect to lose ions above about $5.6 \mathrm{keV}$, while the maximum in the integral for $\langle\sigma \mathrm{v}\rangle$ is found to be at $6.8 \mathrm{keV}$. Thus, significant quenching might be expected.

The simplest estimate of the effect is obtained by adopting $\lambda=R$ as the criterion for lost ions, and truncating the Maxwellian tails at the corresponding energies in performing the $\langle\sigma \mathrm{V}\rangle$ integration, which must now be done using Eqs. (2) or (4), not (5), because the distributions are no longer Maxwe1lian. The c'itoff $E_{c}$ 's obtained are shown in $F i g .1$ for a $1.0 \mu g$ microsphere. The weak tenperature dependence (in $2 n \Lambda$ ) is indicated; the still weaker mass (or density in $\ln \Lambda$ ) dependence is insignificant for 0.1 to $10.0 \mu \mathrm{g}$ masses. Using a best $\mathrm{fit}^{6}$ to empirical data for tre cross-section $\sigma$, we have integrated $<\sigma v>$ numerically for Maxwell distributions cut off at various energies. The resulting $\langle\sigma v>$ curves are plotted in Fig. 2. It is observed that at high $E_{C}$ the several curves saturate to the traditional < ov $>_{\infty}$ values, whereas at smaller $E_{C}$ they fall rapidly and become approximately independent of temperature. This approximate temperature independence is perhi.ps not very surprising because the distributions are all flat at small energies. To obtain a better understanding of the important values of $E_{c}$, than simply that from Eq. (4), we have plotted the $E_{c}$, which reduces $<\sigma v>$ to 0.5 and tc 0.1 of its asymptotic value in Fig. 3. The $(k T)^{2 / 3}$ - dependence for small temperatures is apparent.

For a more sophisticated estimate of the effect of ion losses, we use a Fokker-Planck code based on the work of Rosenbluth, MacDonald, and Judd. ${ }^{7}$ We 


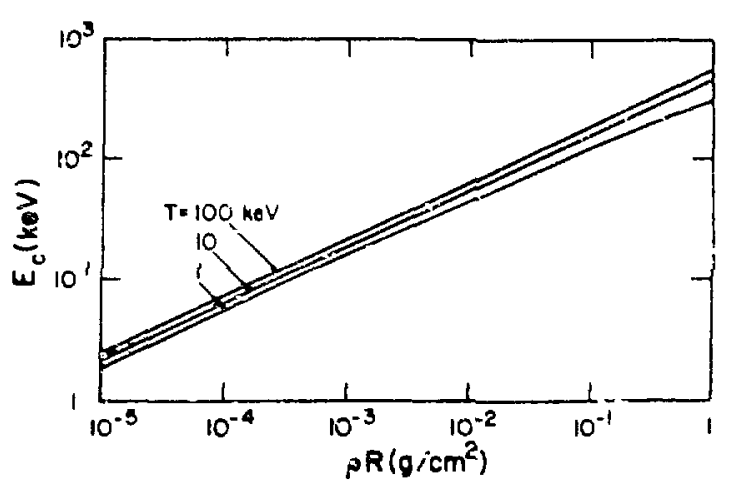

Fig. 1.

Cutoff energy $E_{C}$ for ion loss vs areal mass density $p$.

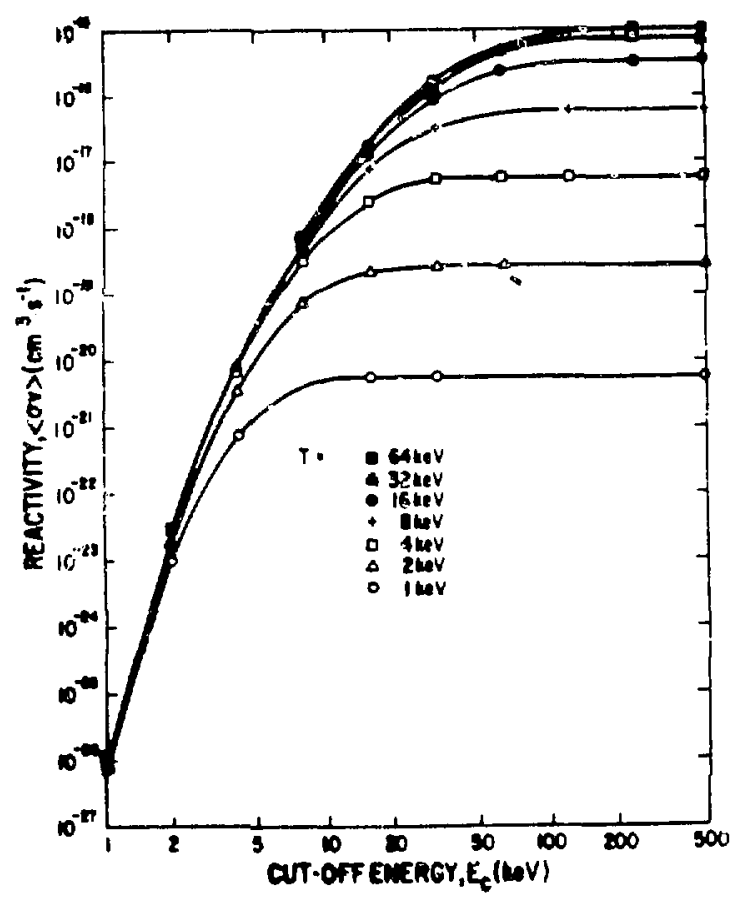

Fig. 2 .

Fusion Reactivity < ov > integrated to cutoff energy $E_{r}$ for various values temperature $\mathrm{T}$.

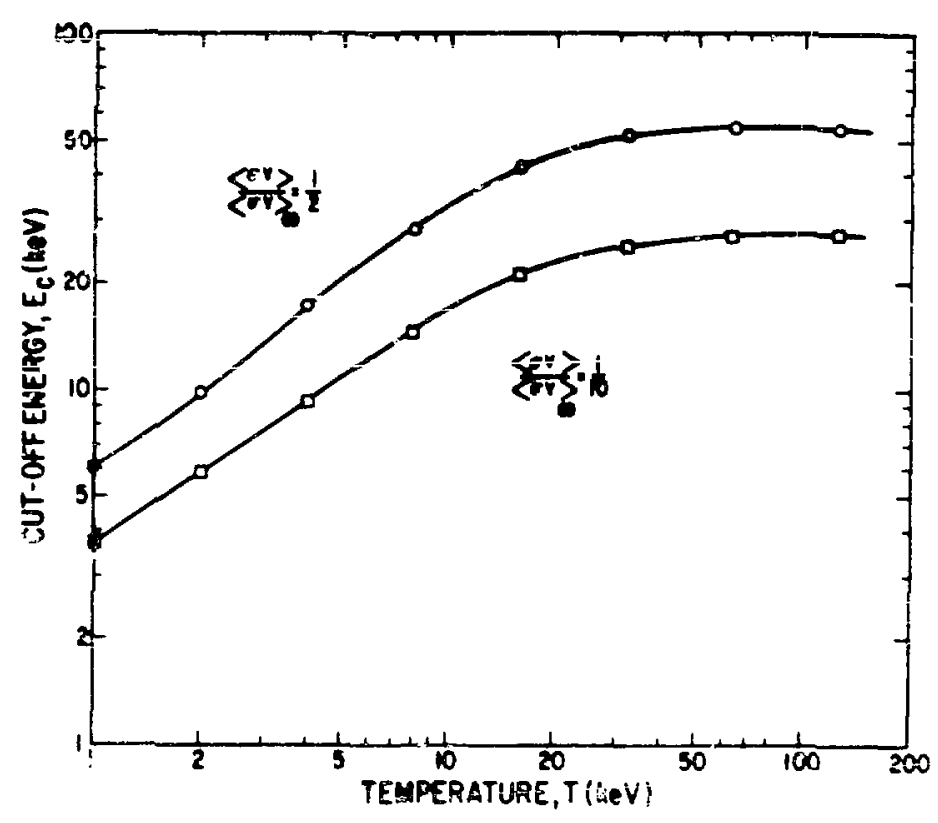

Fig. 3.

Cutoff $E_{c}$ required to reduce the reactivity $\langle\sigma v>$ to 0.5 and 0.1 of its asymptotic value vs temperature $T$. 
recasst their Eqs. (20) and (21) in an obvious way by moving the summation signs to obtain

$$
\frac{1}{\Gamma_{a}}\left[\frac{\partial g_{a}}{\partial t}\right]_{c}=-\frac{\partial}{\partial v_{i}}\left[g_{a} \sum_{b} \frac{m_{a} \cdot m_{b}}{m_{b}} \frac{\partial\left\langle v^{-1}\right\rangle_{b}}{\partial v_{i}}\right]+\frac{1}{2} \frac{\partial^{2}}{\partial v_{i} \partial v_{j}}\left[g_{a} \sum_{b} \frac{\partial^{2}\langle v\rangle_{b}}{\partial v_{i} \partial v_{j}}\right],
$$

with

$$
\begin{aligned}
\Gamma_{a} & =4 \pi e^{4} \text { ln } \Lambda / m_{a}^{2}, \\
\left\langle v^{-1}\right\rangle_{b}(\vec{v}) & =\int d \vec{v}^{\prime} g_{b}\left(v^{\prime}\right)\left|\vec{v}-\vec{v}^{\prime}\right|^{-1,}, \\
\langle v\rangle_{b}(\vec{v}) & =\int d \vec{v}^{\prime} g_{b}\left(v^{\prime}\right)\left|\vec{v}-\vec{v}^{\prime}\right|,
\end{aligned}
$$

where $g$ is the distribution function and all the symbols are as customarily used.

We specialize to the isotropic case by explicitly performing the angular integrations. We then nondimensionalize the equations and perform the indicated differentiations. The variables transform to

$$
\begin{aligned}
& v=v_{\xi} \\
& g=v^{-3} G \\
& t=\left[v^{3} / 2 \pi \Gamma_{I}\right] \tau,
\end{aligned}
$$

where $\xi, G$, and $\tau$ are dimensioniess and $\Gamma_{I}$ is the initial value of $\Gamma_{a}$. (In singlespecies problems without lusses, $\Gamma$ is a constant). In these variables the equations become

$$
\begin{aligned}
\frac{\partial G_{a}}{\partial \tau}= & \left.r_{\bar{a}} / \Gamma_{I}\right] \sum_{b}\left\{G_{a}\left[2\left(\frac{m_{a}}{m_{b}}\right) G_{b}\right]+\frac{\partial G_{a}}{\partial \xi}\left[?\left(\frac{n_{a}}{m_{b}}\right) \xi^{-2} J_{2, b}(\xi)-\frac{2}{3} \xi^{-4} J_{4, b}(\xi)\right.\right. \\
& +\frac{4}{3} \xi^{-1}\left(J_{1, b}(\infty)-J_{1, b}(\xi)\right) \\
& +\frac{\partial^{2} G}{\partial \xi^{2}}\left[\frac{2}{3} \xi^{-3} J_{4, b}(\xi)+\frac{2}{3}\left(J_{1, b}(\infty)-J_{1, b}(\xi)\right)\right]
\end{aligned}
$$


where $J_{n, b}(\xi) \equiv \int_{0}^{\xi} z^{n} G_{b}(z) d z$.

The equations have been encoded in this form. ${ }^{8}$ Two useful auxiliaries are the density

$$
\begin{aligned}
& n_{a}=4 \pi J_{2, a}(\infty) \text {, and mean energy } \\
& \varepsilon_{a} \equiv \frac{3}{2} k T_{a}=\left[\frac{1}{2} m_{a} v^{2}\right] J_{4, a}(\infty) / J_{2, a}(\infty) .
\end{aligned}
$$

A better nordimensionalization, at least for reporting resu'ts, is one using the density-time product ( $n t)$ as the independent variable. This scaling makes the evolution self-similar for problems not involving the loss of particles. An equivalent scaling uses Spitzer's "value for the mean collision time, $t_{c}$,

$$
\nu^{-1} \equiv t_{c}=m^{1 / 2}(3 k T)^{3 / 2} / 8 \times 0.714 \pi n e^{4} \text { en } \Lambda \text {. }
$$

The number of mean collision timar : vt), which is useful for interpretations, and the product $(n t)$, which is useful for applications are simpiy related as

$$
v t=n t \times\left[0.714\left(4 \pi \mathrm{e}^{4} / \mathrm{m}^{2}\right)\left(\frac{3}{2} \mathrm{kT} / \mathrm{m}\right)^{3 / 2} \ln \Lambda / \sqrt{2}\right],
$$

where the sạijare bracket is a constant for many problems.

For DT reactions we carry only an average ion of atomic mass 2.5 and compute the fusion reactivity $<\sigma v>$ by double quadrature over the distribution function $g(v)$, using $E_{q}$. (4). The values of o used are obtained from the five parameter fit of Duane. ${ }^{6}$ Because the evolving function $g(v)$ is a function of (nt) or (vt), so is its quadrature $\langle\sigma v>$. The fractional burnup

$$
f=\int \frac{1}{2} n<\sigma v>d t
$$

is also clearly a function of (nt) or ( $v t)$ for problems with a constant density in. Note that we are assuming $f \ll i$ and ignore fuel depletion. one of our results will be that the ion-tail filling effect is unimpcrtant in cases giving appreciable fractional burnup. 
The Coulomb logarithr. is taken from dewitt ${ }^{9}$. In our computations we have evaluated it with the mean ion energy' $E$ and ion density alone. This is equivalent to neglecting electron shielding and the energy dependence of $\Lambda$, which are probabiy smali errors.

In some models of pellet burn the velocity distribution initially, that is at the instant of collapse, may be thought of as a delta function in speed: ions moving in all directions but with a common speed equal to that at which the shell moved inward. ${ }^{2}$ Hence we have calculated the relaxation of $\langle$ ov $>$ toward its Maxwellian value $<\sigma v>_{M}$. This relaxation of $<$ ov $>$ toward its asymptotic value $<\mathrm{CV}\rangle_{M}$, corresponding to a Maxwellian ion distribution when the ions were all started at (neariy) the same speed, is shown in Fig. 4 for a number of cases. In each case the temperature $T$ is that of the Maxwellian; the initial beam speed is $v=(3 \mathrm{kT} / \mathrm{m})^{l / 2}$. The horizontal lines are the asymptotic values < or $>_{M}$. From the figure we see that the relaxation requires more collision times at the lower energies, because of the steepness of the cross section $\sigma$ at lower energies. The

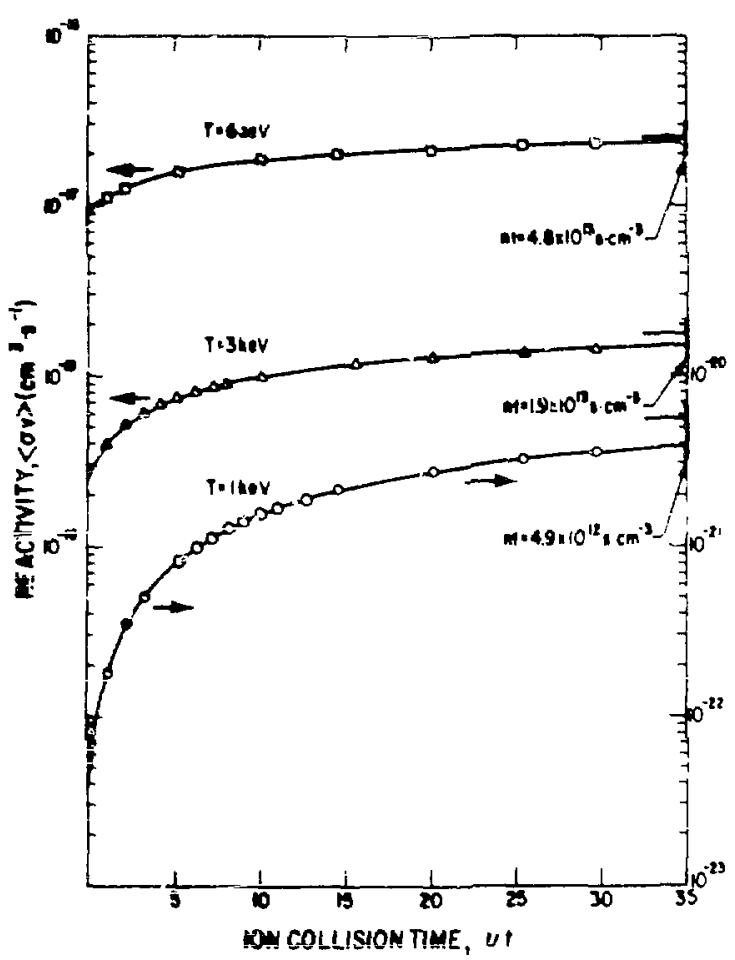

Fig. 4.

Time evolution of the fusion reactivity

- $\mathrm{OV}>$ for various temperatures T. time conversion factors and the initial and asymptotic values of < ov > are listed in Table I.

In Fig. 5 we plot the distribution function $g(v)$ as it relaxes toward the Maxwellian. The plot is for the $T=6-k e V$ case, but is really seif-similar if scaled for different energies. We see that even after nearly 100 mean collision times, the deviation from Maxwe 11 ian is appreciable at 10 times the thermal energy.

The initial delta=functions used here were actually narrow Gaussians in speed with a width equal to $1 / 25$ of the mean speed. Values of $1 / 10,1 / 20$, and $1 / 40$ were also tried to verify that no effects were evident in the results of interest. That is, the beam 
spreads from a "l/25" shape to approximateiy a "1/10" shape in a small fraction of a collision time, $u t \ll 1$.

TABLE I

\begin{tabular}{|c|c|c|c|c|}
\hline $\mathrm{T}(\mathrm{keV})$ & $n t / v t$ & $\left\langle\sigma v{ }_{0}\left(\mathrm{~cm}^{3} / \mathrm{sec}\right)\right.$ & $<\sigma \mathrm{V}\rangle_{M}\left(\mathrm{~cm}^{3} / \mathrm{sec}\right)$ & $\langle\sigma V\rangle_{O} /\langle\sigma V\rangle_{M}$ \\
\hline 1 & $1.40 \cdot 10^{11}$ & $3.98 \cdot 10^{-23}$ & $5.49 \cdot 10^{-21}$ & $7.25 \cdot 10^{-3}$ \\
\hline 3 & $5.49 \cdot 10^{11}$ & $2.38 \cdot 10^{-19}$ & $1.72 \cdot 10^{-18}$ & $1.38 \cdot 10^{-1}$ \\
\hline 6 & $1.35 \cdot 10^{12}$ & $8.48 \cdot 10^{-18}$ & $2.43 \cdot 10^{-17}$ & $3.49=10^{-1}$ \\
\hline
\end{tabular}

In Fig. 6 we show the evolution of the fractional burnup $f$, on linear scales, piotted against iinear scales of time (ut) and also (nt). The plot is

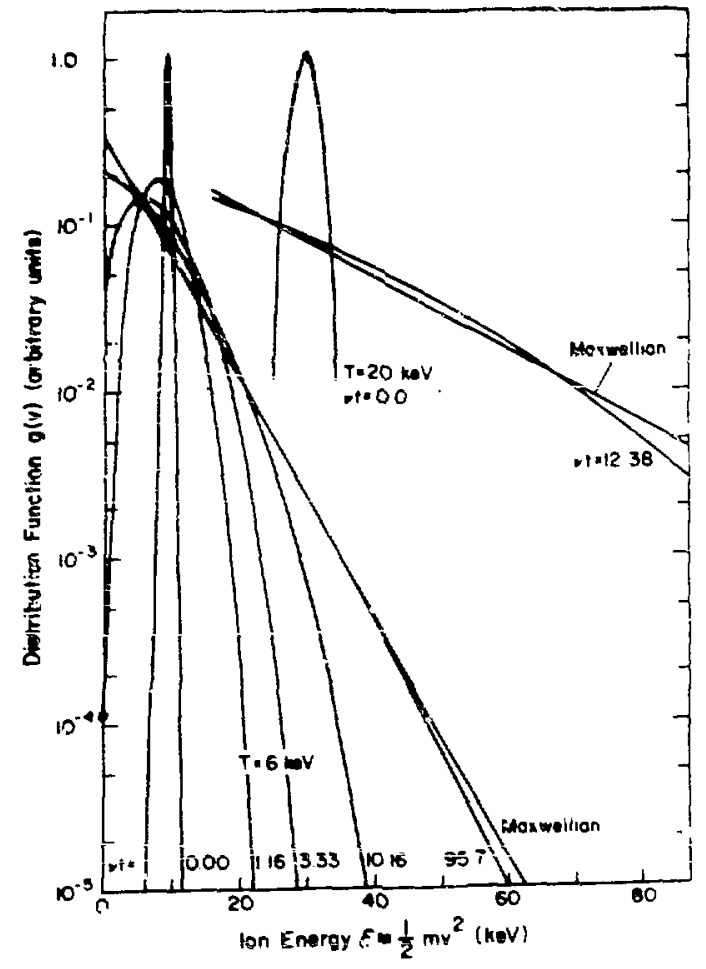

Fig. 5 .

Evolution of the distribution function $g(v)$ vs ion energy $\varepsilon=1 / 2 M v^{2}$. Initial near-delta function distributions were at $T=6$ and $20 \mathrm{keV}$. for the 6-kel case, but illustrates a convenient parameterization of the resuits. Comparing the fractional

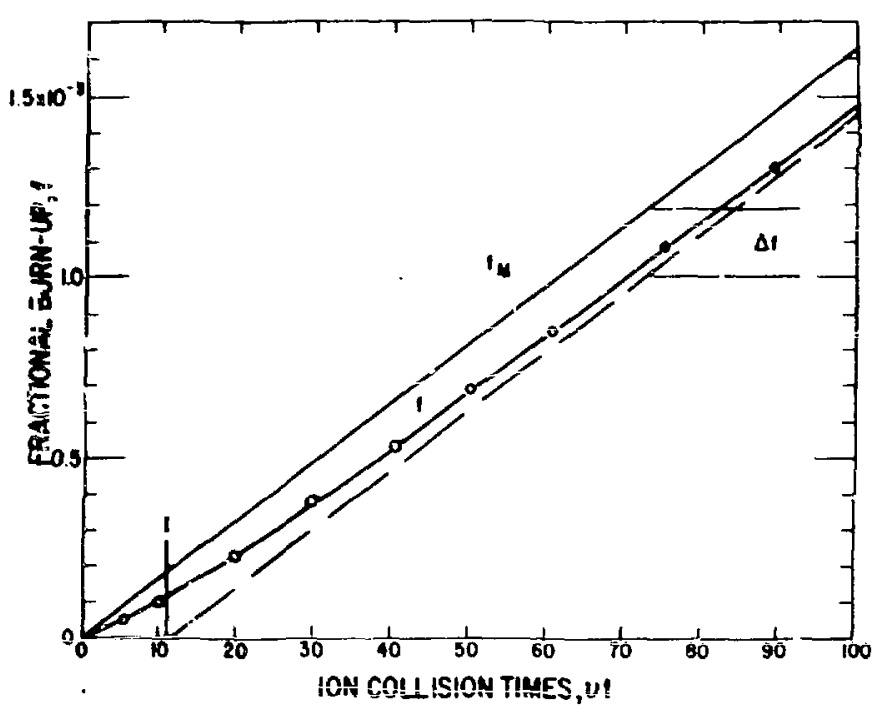

Fig. 6.

Fractionel burnup $f$ for an evolving distribution at $\delta \mathrm{keV}$. 
burnup $f$ with the a priori burnup $f_{M}$ which would have occurred had the Maxwellian < ov $>_{M}$ applied for ail time we note that $f$ becomes parallei to $f_{M}$ us $\langle\sigma y\rangle$ saturates. For probiems with relative?y long burn-times, it is convenient to simply subtract ôf the "lost burniop" if from the burmup computed by the simulation code. This corrects for the code's assuming Maxwellian ion distribution at all times.

Alternatively, we could think of suppressing the start of burn (at the Maxwellian rate) by a relaxation time or induction time

$$
(n t)_{1}=\Delta f / \frac{1}{2}<\text { ov }>_{M},
$$

which is indicated by the intercept " $i "$ in the figurs. Clearly, cases which proceed to large fractional burnup

$$
f>\Delta f
$$

or to large burn-time $t_{B}$,

$$
(n t)_{B} \gg(n t)_{I}
$$

will not need significant corrections.

Computed values of $\Delta f$ and $(n t)_{I}$ are listed in Table II for various temperatures $T$. The comparison of the a priori f froin a hydrodynamics/burn calculation with $\Delta f$ as computed here would seem to be the better test of whether a correction is needed, because of the possibility of bootstrap heating raising $\langle\sigma v>$ with time as the burn ensues. On the other hand, Table II reveals a remarkable constancy in the values of $(n t)_{I}$ for the various temperatures of interest. As a

\section{IABLE II}

$\begin{array}{ccc}\frac{T(k e v)}{1} & \frac{\Delta f}{1.7 \cdot 10^{-8}} & \frac{(n t)_{1}=2 \Delta f /\langle\sigma V\rangle_{M}\left(\mathrm{sec} / \mathrm{cm}^{3}\right)}{6.4 \cdot 10^{12}} \\ 3 & 9.2 \cdot 10^{-6} & 1.1 \cdot 10^{13} \\ 6 & 1.6 \cdot 10^{-4} & 1.5 \cdot 10^{13}\end{array}$


handy ruie of thumb, we conclude that corrections are not required for cases giving $(n t)_{B}$ for the burn much in excess of $10^{12} \mathrm{~cm}^{-3} \mathrm{sec}$.

We can derive a simple approximate formula for the fractional burnup, $f$, which is quite good. For short times, $(n t) \ll(n t)_{I}$, there is only negligible evolution of $<\sigma V>$ from the initial $<\sigma V>_{0}$, thus

$$
f=f_{0} \equiv \frac{1}{2}<\sigma v>_{0}(n t), n t \ll(n t)_{I} .
$$

For long times, (nt) $>>(n t)_{I}$, we have fraciional burnup at the asvmptotic rates $<\sigma \vee>_{M}$ with only the constant $\Delta f$ missing due to the evolution:

$$
f \approx f_{M}-\Delta f \equiv \frac{1}{2}<\sigma V>_{M}\left[(n t)-(n t)_{I}\right], n t>(n t)_{I}
$$

The simplest rational form with these limits is

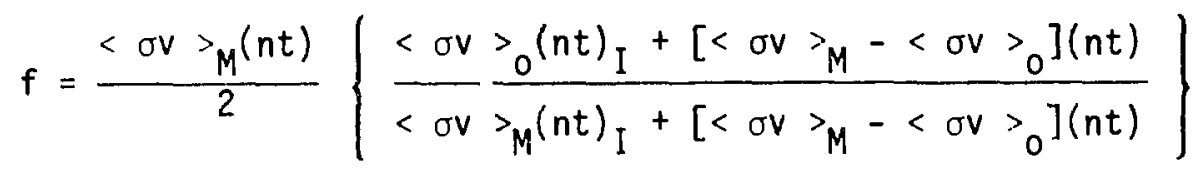

or

$$
f=f_{M}\left\{\frac{2 \Delta f_{0}+f_{M}-f_{o}}{2 \Delta f+f_{M}-f_{0}}\right\}
$$

with the above definitions plus $\Delta f_{0} \equiv \frac{1}{2}<$ ov $>_{0}(n t)_{I}$.

When applied to the data of Fig. 6, this approximation yields results within $1 \%$ of the computed results. These are indisiinguishable from the plotted curve $f$. Although we have not proven the universal applicability of tile convenient form (14), we believe it to te entirely adequate for all applications. It does provide a good fit to the computations reflected in Tables I and II.

Instead of simply assuming a cutoff in the Maxwell distribution, we can calculate the distribution function dynamically starting from either a Maxwell distribution or the single speed distribution used above and including losses. This is accomplished by adding an appropriate loss term to the Fokker-Planck treatment. What is not so clear, however, is now one could treat the bounding conditions: are particles lost, is energy los:, etc.? A complete trea iment would actually allow fluxes of both particles and energy, with the boundary 
conditions being specified as functions of time from a coupled hydrodynamics calculation. Such a major undertaking does not appear justified since we have found the non-Maxwellian effects to disappear at significant compressions ( $\rho R>0.01 \mathrm{~g} \mathrm{~cm}^{-2}$ ).

In the spirit of postcorrections to hydrodynamics calculations that assume Maxwellian distributions, we have performed some ion loss and combined-ion loss and ion evolution-calculations. For the loss term we consider the loss of particles by diffusion from a uniform sphere. Using well-known results from reactor theory, the loss is

$$
\frac{1}{N} \frac{d N}{d t}=\frac{\lambda v}{3 N} \nabla^{2} N
$$

Taking $\frac{1}{N}{ }^{2} \mathrm{~N}$ to be constant over the sphere of radius $\mathrm{R}$, we have

$$
\frac{1}{N} \nabla^{2} N=\frac{\pi^{2}}{R^{2}} \text {, so that } \frac{1}{N} \frac{d N}{d t}=\frac{\pi^{2}}{3} \frac{\lambda v}{R^{2}}
$$

for each group of ions at speed $v$. We have included this term in the computer code, with $\lambda(v)$ evaluated as in Eq. 6. The particles that are lost by this prescription are replaced by multiplying the distribution function by a constant. Otherwise, the Fokker-Planck calculations proceeded as described above.

The decrease of the <ov $>$ value as a function of time for a $1 \mathrm{keV}$ starting Maxwell distribution and several values of $\mathrm{pR}$ is shown in Figs. 7 and 8 . The evolution of the "temperature," defined as $2 / 3$ the mean kinetic energy, that leads to the ion average energy as reduced by the losses is also shown. Comparing the actual $\langle\sigma v\rangle$ to the Maxwell average $\langle\sigma v\rangle$ at this temperature shows that the predominant effect is energy loss rather than the deviation of the tail of the distribution from Maxwellian. The calculated distribution for a case with sinall oR when $\langle\sigma V\rangle_{M} /\langle\sigma V\rangle$ is about 4 , but $\langle\sigma V\rangle_{M}$ itself has dropped by a factor of 3000 is compared to a Maxwellian in Fig. 9 and shows a substantial deficit in the tail at the energy that makes maximum contribution to $\langle\sigma \mathrm{v}\rangle$ in the Maxwellian. The double integral reduces the effect on <ov> substantiaily. We have aiso run several cases starting with all the ions at the same

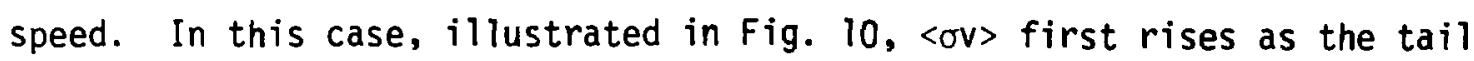
fills out and then drops as energy is lost from the distribution. The 


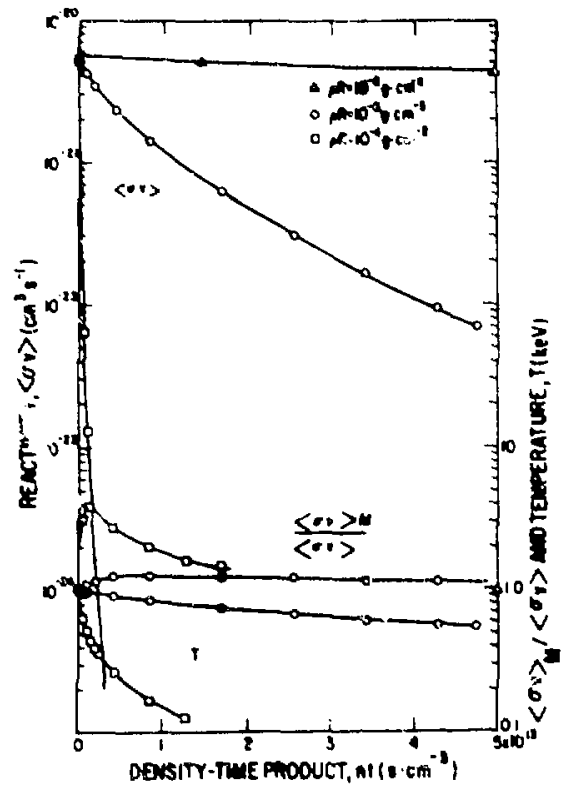

Fig. $\%$

Time evolution of fusion reactivity $\langle\sigma \checkmark\rangle$ and the mean energy $T$ for an initial Maxwell distribution at $T=l \mathrm{keV}$ including ion losses for $\rho R=10^{-4}, 10^{-3}$, and $10^{-2} \mathrm{gcm}^{-2}$.

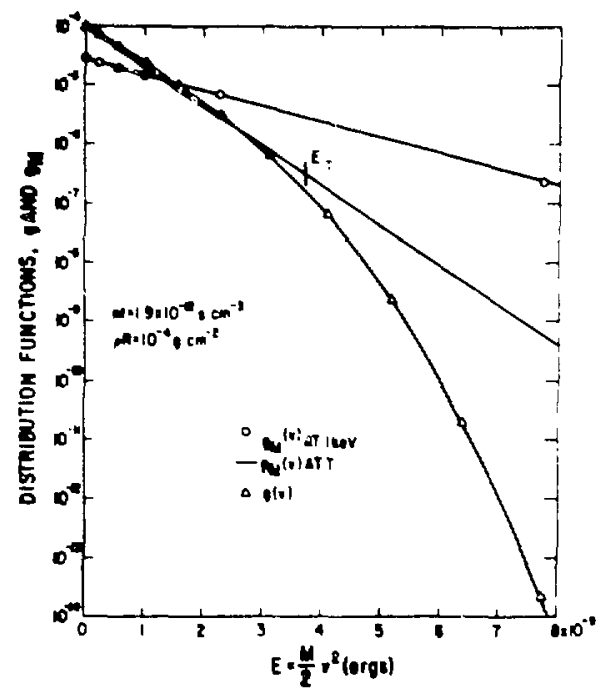

Fig. 9.

Distribution function $g(v)$ at $\mathrm{nt}=1.9 \times 10^{12} \mathrm{~s} \mathrm{~cm}^{-3}$ for the case of Fig. 8. If the Maxwellian distribution at $T=0.4 \mathrm{keV}$ is truncated at $E_{T}=3.7 \times 10^{-}$ ergs, the resulting reactivity $\langle\sigma v\rangle$ equals that for the relaxing distribution $g(v)$.

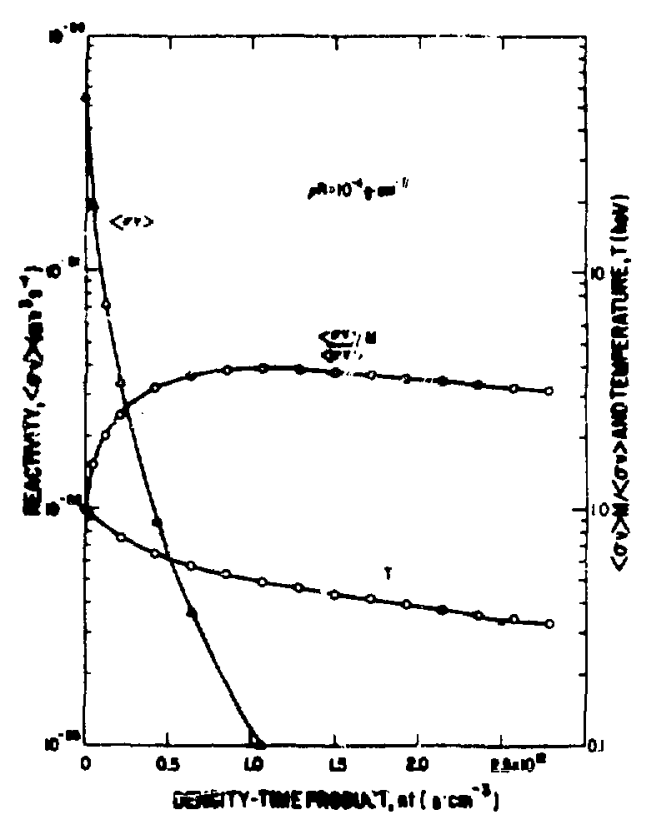

Fig. 8.

Earliest time evolution of $\rho R=10^{-4} \mathrm{gcm}^{-2}$ case from Fig. 7, plotted on an expanded scale.

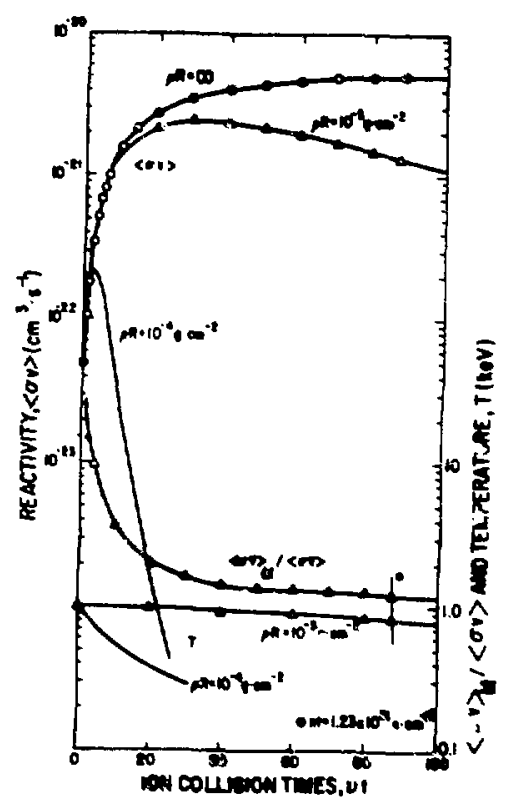

Fig. 10 .

7 ime evolution of fusion reactivity <ov> and mean energy $T$ for an initially near-delta function at $T=? \mathrm{keV}$, with ion losses. Compare with Figs. 7 and 8. 
$O R=10^{-2} \mathrm{~g} \mathrm{~cm}^{-2}$ and the no-loss results show only insignificant differences. That is, $\rho R=10^{-2} \mathrm{~g} \mathrm{~cm}^{-2}$ is effectively thick as we have already concluded. As the tail fills out, from the initial delta-function toward regions of $v$ with significant particle loss, we notice the values of <ov $\rangle$ begin to $d^{-} p$. The $\rho R=10^{-4} \mathrm{~g} \mathrm{~cm}^{-2}$ case shows an actual decline in $\langle\sigma \mathrm{V}\rangle$ with time from a maximum at about 10 collision times. This is accompanied by significant cooling, as may be seen from the values of $T \equiv M<v^{2}>/ 3 k$, which are also plotted. Had the problem been done with the distributions renormalized to maintain a constant $T$, the results would clearly be different. In a physical problem there would, of course, be an important heat loss and a decrease in fuel pressure; the se would be important to the hydrodynamics.

The fractional burnup values for the 3-keV case are shown in Fig. 11. For the very thin $p R=10^{-4} \mathrm{~g} \mathrm{~cm}^{-3}$ case, the fractional burnup becomes nearly

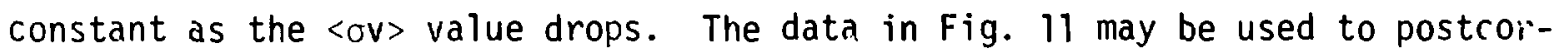
rect hydrodynamics-burn calculations in the same way as that in Figs. 3 or 4 . Simple bare pellets are known to disassemble in an expansion time ${ }^{l}$

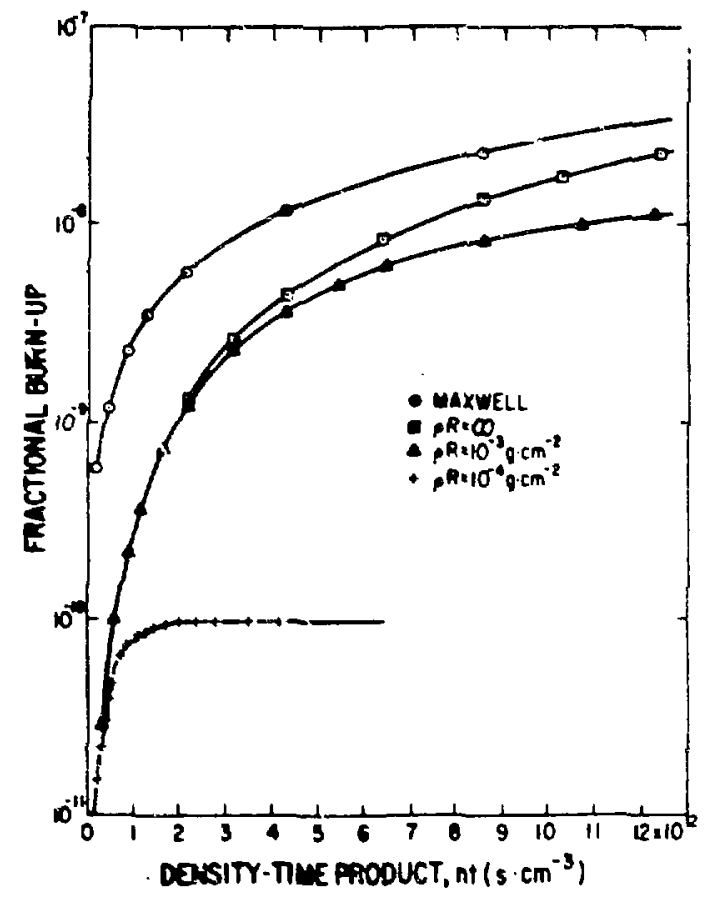

Fig. 11.

Fractional burnup for the evolving distribution with ion losses from Fig. 10.

$$
\tau_{e}=R / 4 C_{s}, \quad C_{s}=\text { sound speed. }
$$

For 1.0-hg DT spheres this works out to approximately

$$
\begin{aligned}
& \tau_{e}=3.410^{-12}(\rho R \quad T)^{-1 / 2} \\
& (i, \rho, R \text { in cgs units, kT in keV). }
\end{aligned}
$$

In all cases the disassembly is so racid as to occur before the fractional burnup deviates significantly from the thick ( $\rho R \equiv 10^{-2} \mathrm{~g} \mathrm{~cm}^{-2}$ ) result. That is, in these probiems the truncation of the distribution by ion loss will have no effect. In structured systems, however, the confinement times may be considerably ionger. Deviations from the thick-case results, if they are 
important, will be primarily due to energy losses rather than deviations from ilaxwell distributions.

Having explored the ion loss effect, the time evoiution of the distributions and the combined problem, we feel that we understand the observed yields, which are sumewhat below a priori (Maxwellian) estimates. ${ }^{10}$ It is possible to construct hydrodynamics-burn computer codes that take proper account of these effects, using the methods outlined here. However, in view of the effects becoming unimportant above $\rho R=0.01 \mathrm{~g} \mathrm{~cm}^{-2}$, the effort does not appear justified.

\section{REFERENCES}

1. G. S. Fraley, E. J. Linnebur, P. J. Mason, and R. L. Mo.se, "Thermonuclear Burn Characteristics of Compressed Deuterium-Tritium Microspheres," Phys. Fluids 17, 474 (1974).

2. J. S. Clark, H. N. Fisher, and R. J. Mason, "laser-Driven Implosion of Spherical DT Targets to Thermonuclear Burn Conditions," Phys. Rev. Lett. 30, 89, (1973) and 30, 249 (1973).

3. J. Nuckolls, L. Wood, A. Thiessen, and G. Zimmerman, "Laser Compression of Matter to Super-High Densities: Thermonuclear (CTR) Applications," Nature (London) 239, 139 (1972).

4. L. Spitzer, "Physics of Fully Ionized Gases," (Interscience, New York, 1st Edition 1952, 2nd Edition 1962).

5. D. B. Henderson, "Burn Characteristics of Marginal Deuterium-Tritium Microspheres, "Los Alamos Scientific Laboratory report LA-5603-MS, (May 1974). Phys, Rev. Lett. 33, 1142 (1974).

6. B. H. Duane, Battelle Northwest Laboratory, unpublished data, 1972.

7. M. N. Rosenbluth, W. M. MacDonald, and D. L. Judd, "Fokker-Planck Equation for inverse-Square Force," Phys. Rev. 107, 1 (1957).

8. W. M. MacDonald, M. N. Rosenbluth, and W. Chuck, "Relaxation of System of Particles with Coulomb Interactions," Phys. Rev. 107, 350 (1957).

9. H. deWitt, in Lectures in Theoretical Physics, Vol. IX, Part C, (Brittin, eds. Gordon and Breach, 1967) p. 621.

10. H. Brysk and P. Hammerling, "DT Fusion with Microshells," Phys. Rev. Lett. 34,502 (1975). 